\title{
El mercado de trabajo argentino durante la post convertibilidad. La dinámica de la actividad económica, del empleo y el ingreso en la Argentina
}

\section{The Argentine labor-market during post-convertibility. The dynamics of economic activity, employment and income in Argentina}

ARTÍCULO

\section{Héctor Gabriel Bazque}

Universidad Nacional de Quilmes, Argentina. Contacto: hbazque@unq.edu.ar

Recibido: junio de 2021

Aceptado: agosto de 2021

\section{Resumen}

Análisis de la dinámica del trabajo y del ingreso en Argentina durante el período de post convertibilidad. Primero se efectúa un abordaje teórico de la cuestión del equilibrio en el mercado de trabajo y la distribución del ingreso. Luego se repasa la dinámica de los principales indicadores económicos del país.

El intento de implementar un modelo de desarrollo basado en la hipótesis neoclásica de distribución del ingreso fracasó nuevamente en Argentina durante el gobierno de Mauricio Macri. Una vuelta a la hipótesis keynesiana, con intervención estatal y estímulo al desarrollo de la capacidad competitiva del entramado productivo parece ser un camino más adecuado. Por lo tanto, es importante generar las condiciones para el establecimiento de un nuevo ciclo de crecimiento económico, sin descuidar la capacidad adquisitiva y el bienestar de la fuerza de trabajo.

Palabras clave: Empleo, distribución del ingreso, instituciones, economía.

\begin{abstract}
Work and income dynamics analysis in Argentina throughout the post-convertibility period. First, a theoretical approach is applied to assessed labor market equilibrium and income distribution. Then, the dynamics of the main economic indicators of the country are analyzed. The attempt to applied a development model based on the neoclassical hypothesis of income distribution, failed again in Argentina during the government of Mauricio Macri. A return to the
\end{abstract}


Keynesian hypothesis, with state intervention and encouragement to the development of productive network competitiveness seems to be a more suitable path. Therefore, it is important to create the conditions for the establishment of a new economic growth cycle, without neglecting the purchasing power and welfare of the workforce.

Keywords: Employment, income distribution, institutions, economy.

\section{Introducción}

En Argentina, desde la salida de la convertibilidad, las discusiones sobre economía y desarrollo se encuentran constantemente entrelazadas con la cuestión de la distribución del ingreso. El criterio de política adoptado en este tema define las relaciones sociales que se dan en la producción, el rol de las instituciones y el sostenimiento del proceso de acumulación, determinando tanto la cantidad como la calidad de empleo.

De fondo, la discusión que se plantea es sobre distribución del ingreso entre clases sociales, referida a qué tipo de política se debe promover desde el sector público. Habitualmente la tensión se da entre dos miradas económicas alternativas, aquella más relacionada con la perspectiva ortodoxa (o neoclásica) y otra más afín al enfoque del Estado de bienestar keynesiano. Probablemente, temas como la transformación de la estructura productiva, el desarrollo de proveedores locales de insumos industriales intermedios y el mejoramiento de la dinámica comercial (interna y externa) constituyan objetivos compartidos por todos los sectores de la sociedad argentina, pero identificar y consensuar cuál es el camino socialmente más justo para lograrlo, parece algo mucho más difícil de definir.

El inicio y culminación de los ciclos de acumulación de cada momento, que impactan en forma determinante sobre la actividad económica en general, y el mercado de trabajo en particular, son definidos por las instituciones que se construyen socialmente, regulando tanto la producción como las relaciones que se establecen entre los actores involucrados en dicho proceso. Entre trabajadores y empresas existe una puja con intereses contradictorios, en donde son aspectos centrales la remuneración, la intensidad del trabajo y su calidad.

Con el fin de la convertibilidad en Argentina se inició un nuevo ciclo de acumulación, impulsado y sostenido bajo una nueva configuración institucional. Este modelo logró superar la crisis financiera internacional del 2008, pero desde 2011 en adelante comenzó a mostrar algunos síntomas de debilitamiento, que en 2015 impuso el cambio del signo político al frente 
de la gestión del gobierno nacional. Con ello se definieron nuevas reglas del juego (o instituciones) y un claro intento de establecer un modelo de acumulación diferente.

El objetivo de este documento es realizar un análisis de la dinámica del trabajo y del ingreso en Argentina durante el período de post convertibilidad. Para ello, primero se efectúa un abordaje teórico de la cuestión del equilibrio en el mercado de trabajo y la distribución del ingreso. Luego se repasa la dinámica de los principales indicadores económicos del país, para finalizar explorando los principales dilemas durante el contexto actual.

\section{Marco Teórico}

La teoría económica ortodoxa construye un modelo matemático a partir del cual se procura explicar la forma en que se alcanza el equilibrio en el mercado de trabajo. Desde esta perspectiva, la demanda de trabajo se encuentra constituida por las empresas que requieren emplear personas (y su fuerza de trabajo) para echar a andar la maquinaria productiva. Es decir, se trata de una extensión de la teoría del productor neoclásico, donde el empresario debe retribuir al trabajo según su aporte a la producción. Contrariamente, la oferta surge del trade-off entre trabajo y ocio, ya que ambos brindarían satisfacción (o utilidad) a las personas. El trabajo permite acceder a un ingreso, y mediante ello acceder al consumo de bienes. Entonces, la predilección por una u otra dependerá exclusivamente de cuál de las dos aporte mayor grado de satisfacción; es decir, de su utilidad marginal. En este sentido, al igual que con la demanda, la explicación de la oferta de trabajo es una extensión de la teoría del consumidor.

Este enfoque entiende al equilibrio de mercado de trabajo como al de cualquier otro mercado de bienes, identificando un precio (salario real) y una cantidad de trabajo. A partir de ello, ante cualquier situación de desequilibrio, el mercado resuelve el problema con un ajuste del salario real, garantizando siempre el pleno empleo. Es decir, para este enfoque no queda lugar para el desempleo involuntario. En tal caso, los causantes del desempleo son los propios trabajadores, que no aceptan que sus salarios disminuyan a niveles de equilibrio (Costa et al., 2003; Nicholson, 2008).

La obra de Keynes surge a partir de la incapacidad de la teoría neoclásica para explicar el desempleo involuntario. Desde este enfoque, el ajuste en el mercado de trabajo no resulta automático como proponían Pigou y los economistas ortodoxos. Para Keynes la creación (o demanda) de fuentes de empleo no dependen del nivel de los salarios, sino de la eficiencia marginal del capital (junto a la tasa de interés y la demanda efectiva), que es la que promueve el incremento de la inversión. Así, las empresas son las que definen el número de empleos, 
mientras que el salario (pero esta vez el nominal) es definido exógenamente por los convenios colectivos de trabajo. Los trabajadores y los sindicatos no tienen forma de definir el salario real, ya que el control del nivel de precios se encuentra fuera de su alcance y capacidad de acción (Astarita, 2018; Chamorro, 2011; Pérez y Neffa, 2006).

Desde la perspectiva Keynesiana, el nivel de empleo depende del ingreso nacional, que es el que define el nivel de actividad y producción de una economía (demanda y oferta agregada) y estimula a los animal spirits de los empresarios, pero no de la flexibilidad del salario nominal, que normalmente tiene a ser inflexible a la baja.

En Argentina, durante gran parte del período de post convertibilidad, la discusión sobre empleo e ingresos incorpora recurrentemente a la inflación como principal tema de discusión, imposibilitando el acuerdo de miradas entre la clase trabajadora y los empresarios, quienes quieren evitar el deterioro de sus niveles de ingreso en cada instancia de negociación paritaria. Por un lado, los trabajadores buscan sostener (y si fuera posible, mejorar) su capacidad de compra mediante un incremento del salario nominal; mientras que por el otro, los empresarios, que consideran a los salarios como un costo, buscarán que se incrementen lo menos posible para sostener su margen de ganancia. Sin embargo, es posible pensar que ante una situación de caída de la tasa de ganancia como consecuencia de un incremento salarial, su "reacomodamiento" se dará mediante un ajuste de precios como consecuencia de la asimétrica distribución de poder entre empresarios y trabajadores. Así, el sostenimiento de una mejora salarial dependerá de la fuerza sindical, por un lado, y de las políticas públicas o mecanismos de regulación salarial, por el otro (Barrera Insua y Fernández Massi, 2017).

Este punto abre la discusión sobre qué tipo de política de distribución del ingreso se debe promover desde el sector público, que habitualmente se define pendularmente entre las hipótesis de distribución del ingreso ortodoxa (o neoclásica) y keynesiana, de acuerdo a las convicciones del gobierno que se encuentre ejerciendo la gestión pública.

En este sentido, para la "hipótesis neoclásica", que adhiere a la Ley de Say, donde la oferta crea su propia demanda, el crecimiento de la producción a través del incremento del ahorro y la inversión -mediados por la tasa de interés- es compatible con altos niveles de inequidad a favor del capital en la distribución del ingreso. Por ello, una distribución del ingreso más equitativa es entendida como el resultado de una economía con presencia de grandes distorsiones; es decir, producto de la intervención estatal. Un ejemplo de esto (supuestamente distorsivo) lo representan los acuerdos institucionales que garantizan la realización de negociaciones colectivas de trabajo. Para la "hipótesis keynesiana", la mala distribución de los ingresos, al restringir el consumo, aumenta la necesidad de inversiones para incrementar la demanda y con ella la producción (Porta y Bianco, 2011). Desde este punto de vista, en 
situaciones de desequilibrio, la intervención del Estado (garantizando tanto el sostenimiento de los niveles salariales como de los precios) resulta fundamental para promover la inversión y garantizar la continuidad del empleo.

Entonces, considerando la forma en que se estructura la teoría económica ortodoxa, se entiende su incapacidad para mejorar el grado de bienestar de la sociedad. Esta lógica hizo que la teoría económica ortodoxa no pudiera explicar situaciones donde personas que desean trabajar no consiguen empleo, como lo ocurrido en 1929. Luego, con el surgimiento del Estado de Bienestar Keynesiano, se legitimó a la intervención estatal para orientar la producción y la generación de empleo; siendo importante destacar que existía una estrecha vinculación entre los patrones de consumo homogéneos de la época y el método de producción fordista, que se articulaban correctamente con las políticas de corte keynesiano. Sin embargo, estas tienen sentido si son acompañadas por inversiones de la estructura productiva nacional, que garanticen niveles de competitividad lo suficientemente importantes como para alcanzar la obtención de saldo comercial positivo.

La crisis del Estado de bienestar keynesiano durante la segunda parte del siglo XX puede ser explicada tanto desde una lectura macroeconómica como microeconómica. Desde el enfoque "macro", la crisis del sistema surgió principalmente por su incapacidad para resolver la estanflación, que aquejaba a gran parte del mundo occidental, desde la segunda parte de los años sesenta; mientras que desde la "micro", comenzó a verificarse que las políticas de incentivo al consumo comenzaban a resultar poco efectivas si no eran acompañadas por mejoras en el plano productivo (Brenner, 1999). Esto último provocó que la preocupación referida a cómo mejorar los estándares de competitividad comenzara a hacerse cada vez más fuerte; de lo contrario, las políticas públicas de estimulo al consumo terminaban significando una transferencia de recursos hacia el exterior, hacia países con estructuras productivas más competitivas.

A partir de esta noción, se puede advertir que la efectividad de la intervención estatal en la economía en general, y en la política salarial en particular, encuentra límites allí donde no es validada por la capacidad de acompañamiento del aparato productivo. Además, la lógica de funcionamiento del sistema capitalista comenzó a cambiar fuertemente sobre las últimas décadas del siglo pasado, complejizando aún más el panorama. El mundo fue testigo de un importante proceso de apertura y avances en las tecnologías de comunicación e información (TICs), que en conjunto implicaron mayor movilidad del capital financiero y facilidades para operar en casi todos los mercado del globo. Además, la emergencia del toyotismo significó el surgimiento de un nuevo paradigma productivo que modificó aún más el concierto internacional. En resumen, el funcionamiento del mundo cambió y ello demandó la creación de 
nuevas reglas del juego; que puede ser entendida como la necesidad de nuevas formas institucionales.

Desde la perspectiva de la escuela regulacionista francesa, con fuerte influencia de las nociones keynesianas y marxista (entre otros ${ }^{1}$ ), los procesos de acumulación capitalista no se "autorregulan", necesitan de un marco institucional para garantizar su reproducción en el tiempo (Gajst, 2010). Por ello, las formas institucionales son las que cumplen la función de regular las relaciones sociales en cada ciclo o régimen de acumulación. La ruptura de dichas relaciones son las que generan las crisis. Según Boyer, la relación salarial, la inserción en el sistema internacional y el Estado son algunas de las formas institucionales que regulan los ciclos económicos (otras dos son el "régimen monetario" y la "competencia"), ya que desde esta mirada, el Estado se traduce en un conjunto de reglas y regularidades que sostienen la continuidad de los ciclos (Boyer, 1992).

Debido a la separación entre los trabajadores y los medios de producción, las formas institucionales surgen para atenuar las contradicciones propias del modo de producción capitalistas. En este sentido, es importante tener en cuenta que la relación salarial no implica sólo cuestiones relacionadas con la retribución a la mano de obra, sino también con el desarrollo de las tecnologías del trabajo y la organización de sus tiempos (Jofré y Ferreyra, 2010). De este modo, el establecimiento y sostenimiento de formas institucionales acordes a las necesidades del ciclo de acumulación vigente garantiza su continuidad.

Hasta aquí podemos ver que según el enfoque adoptado, en relación al funcionamiento del mercado de trabajo y la distribución del ingreso, se definen diferentes opiniones y propuestas para la implementación de política pública. A partir de las acciones implementadas desde el Estado, entendidas desde el enfoque regulacionista como las formas institucionales que encuadran los ciclos de acumulación, la actividad económica mostrará diferentes dinámicas.

A continuación se ponen a prueba las hipótesis y los postulados presentados anteriormente con las decisiones de política económica tomadas desde el gobierno y la dinámica que mostraron los indicadores de desempeño de la economía argentina durante los años posteriores a la salida de la convertibilidad. El objetivo es ver cuál fue la respuesta del mercado frente a enfoques de política pública tan diferentes, como los aplicados por los gobiernos kirchneristas y macrista. Además, se propone pensar las tendencias de cambios del modo de producción internacional y su impacto para el trabajo y la distribución del ingreso nacional.

\section{La dinámica del empleo en Argentina durante la Post Convertibilidad}

\footnotetext{
${ }^{1}$ Como Kalecki, los autores poskeynesianos y el institucionalismo norteamericano.
} 
Con el inicio del Plan de Convertibilidad se definieron una serie de objetivos que apuntaban a modificar las características del mercado de trabajo argentino. Básicamente se buscaba asegurar la movilidad a bajo costo de la fuerza de trabajo para promover transformaciones en la estructura organizacional acordes al nuevo modelo económico (Marticorena, 2005). Hacia fines de 1992 el Ministro de Economía, Domingo Cavallo, anunciaba que se operaría a partir de ajustes sobre aportes y contribuciones patronales, modificando la Ley de contrato de trabajo y el sistema de previsiones para flexibilizar la relación laboral y disminuir el costo salarial. En cuanto a la relación salarial, se desmantelaron los fundamentos básicos del convenio como referencia para la negociación salarial, se abrieron estratos de negociaciones desagregados (Decreto 470/93) y la fijación de techos implícitos para el incremento como consecuencia del Decreto 1334/91, que condicionaba la negociación a la evolución de la productividad. Además, durante este período se daba una suerte de distorsión de la composición salarial como consecuencia de la constante complementación de la remuneración básica con adicionales, premios y extras.

Desde esta perspectiva, el modelo económico aplicado durante los años de vigencia de la Convertibilidad procuraba mejorar las condiciones de competitividad de la oferta local mediante la aplicación de política económica ortodoxa. Con ello se aseguraría tanto el empleo como el consumo de bienes y servicios a bajo precio. Sin embargo, debido a las condiciones instauradas tanto en el plano macroeconómico (Tipo de cambio fijo, apertura comercial, endeudamiento y privatizaciones, etc.) como en el mercado laboral, el resultado del modelo fue la reducción de la tasa de empleo, la "desalarización" y terciarización de la población ocupada, que se impusieron incluso durante los períodos de crecimiento económico de la convertibilidad (Beccaria, 2007; Gómez, 1997; Lindemboim, 2001).

En el período que va desde la salida de la convertibilidad hasta el inicio de la pandemia COVID-19, pueden identificarse tres períodos: una primera etapa de recuperación macroeconómica, que va desde 2003 hasta 2007; una segunda etapa de mayor intervención estatal ejercida con miras a prolongar el ciclo económico, que va desde 2008 hasta fines del 2011; y finalmente una larga etapa de agotamiento, que se inicia a partir del 2012 y se encuentra constituida por dos sub-período: por un lado, uno donde se intentó ejecutar una administración estatal de "sintonía fina"; por el otro, un nuevo experimento neoliberal, donde se otorgó al mercado la responsabilidad de asignar recursos económicos y distribuir ingresos.

\section{1. ¿De dónde venimos? Desde la salida de convertibilidad hasta el 2007}

Con la salida de la convertibilidad, resultó necesario definir un nuevo rumbo para una economía que había atravesado la peor de sus crisis de su historia. Luego de un breve 
período de transición (gobierno de Eduardo Duhalde), la reorientación llegará con el gobierno de Néstor Kirchner. Las principales líneas de acción de este gobierno fueron: i) el desendeudamiento, ii) la construcción de un nuevo enfoque en las relaciones internacionales, poniendo a Brasil, China y Venezuela en el centro de escena, y iii) el impulso al mercado interno y a la industrialización, mediante la promoción de exportaciones y sustitución de importaciones. El principal problema durante este gobierno fue la inflación, que se encontraba latente desde la devaluación que significó salida de la Convertibilidad. Sin embargo, el impacto inicial generó una caída del costo laboral real, a la vez que mejoró la competitividad tanto de las empresas exportadoras como de las que compiten internamente con importaciones. Así, se dio un proceso de mejora salarial se debió más al aumento en el volumen empleo, gracias a las nuevas condiciones macroeconómicas, que al resultado de un movimiento sindical o de una definición de política pública específica (Pérez y Neffa, 2006).

\begin{tabular}{|c|c|c|c|c|c|}
\hline & 2003 & 2004 & 2005 & 2006 & 2007 \\
\hline PBI & $8,8 \%$ & $9,0 \%$ & $8,9 \%$ & $8,0 \%$ & $9,0 \%$ \\
\hline Tasa de actividad & $45,6 \%$ & $45,4 \%$ & $45,9 \%$ & $46,1 \%$ & $46,3 \%$ \\
\hline Tasa de empleo & $37,4 \%$ & $38,9 \%$ & $41,3 \%$ & $42,1 \%$ & $41,7 \%$ \\
\hline Tasa de desocupación & $17,8 \%$ & $14,4 \%$ & $10,1 \%$ & $8,7 \%$ & $9,8 \%$ \\
\hline Empleo no registrado & $49,0 \%$ & $47,9 \%$ & $46,2 \%$ & $43,2 \%$ & $40,4 \%$ \\
\hline
\end{tabular}

Fuente: Valores promedio. Encuesta Permanente de Hogares, INDEC.

Con la recuperación económica, mejoraron los indicadores del mercado laboral en argentina: creció la tasa de empleo; en principio por la implementación de planes sociales, pero a partir de 2005 por el crecimiento del PBI. En el mismo período también se observó cierta mejora en lo que respecta al problema del trabajo no registrado (Neffa, 2012).

Sin embargo, además de la implementación de programas sociales (como por ejemplo el "Plan Jefes y Jefas de Hogar Desocupados"), el papel del Estado durante esta etapa fue regular las condiciones de la relación salarial. Durante este período se impulsaron cambios en la regulación del mercado de trabajo, principalmente en lo referido a relaciones laborales y negociación colectiva. En 2004 se derogó la Ley 25.250 (“La Banelco”) y se sancionó la Ley de Ordenamiento Laboral (Ley 25.877), a partir del cual se dio un proceso de expansión de la negociación colectiva, además de la convocatoria al "Consejo nacional del Empleo, la Productividad y el Salario Mínimo Vital y Movil”, a partir del Decreto 1095/2004 que convocó al Consejo creado por la Ley 24.013 de 1991, inactivo por casi un decenio (Anigstein, 2012). 
Además, desde el gobierno se aplicó todo un conjunto de medidas con miras a recomponer la relación salarial luego de la crisis de 2001, desde aumentos salariales mediante la aplicación de sumas fijas no remunerativas, pasando por la definición de un salario mínimo vital y móvil (SMVM) para señalizar y provocar el incremento salarial en el empleo no registrado, hasta la modificación del marco legal para la negociación colectiva. Tanto la nueva dinámica del contexto macroeconómico, como las medidas implementadas desde el gobierno, posibilitaron una importante recuperación salarial, que llevó incluso a niveles superiores al de la Convertibilidad (Maito, 2019). Sin embargo, todas estas medidas operan principalmente sobre el trabajo registrado ${ }^{2}$, con menos efectividad para resolver las cuestiones en el empleo no registrado, heredados desde la década de los noventa (Barrera Insua y Fernández Massi, 2017; Cortés, 2012).

Hacia 2007 ya se advertía que la dinámica macroeconómica de post-convertibilidad no había logrado modificar el perfil productivo y exportador del país. Ante este panorama, el incremento sostenido de los precios al consumidor comenzó a hacer sentir los reclamos en procura de una recomposición salarial (Barrera Insua y Fernández Massi, 2017; Dabat, 2012).

En consecuencia, la recuperación económica luego de la salida de la convertibilidad tuvo resultados muy positivos en el mercado de trabajo y el empleo. Sin embargo, su dinámica no fue lo suficientemente intensa como para alcanzar una plena recuperación del salario real y resolver estructuralmente el problema de la informalidad, problemas que se venían arrastrando desde décadas anteriores. Estas cuestiones, además de un conjunto de aspectos sociales por resolver, fueron definiendo la agenda de política pública para el período siguiente (Beccaria, 2007; CENDA, 2007; Delfini, 2016).

\subsection{8-2011: Mayor intervención estatal para prolongar el ciclo económico}

A la vez que el ciclo económico comenzaba a mostrar algunos signos de desaceleración, en 2008 se desata una profunda crisis financiera internacional. A diferencia de lo ocurrido anteriormente en la historia económica argentina, esta crisis encuentra al país mejor posicionado en el plano financiero, ya sea por su reducido grado de morosidad, por contar con un sistema financiero más sólido y/o por el menor grado de dolarización de la economía (Abeles, 2009). Mientras que la salida de la convertibilidad posibilitaba tener control sobre política monetaria, la cancelación anticipada de la totalidad de la deuda con el FMI a comienzos del 2006, brindaba al gobierno argentino libertad para la gestión de política económica. Estas circunstancias morigeraron el impacto de la crisis y permitieron hacer frente

\footnotetext{
${ }^{2}$ Hacia fines del año 2012 el 34,6\% de los trabajadores se encontraban bajo esta misma situación.
} 
al negativo escenario internacional mediante el despliegue de todo un conjunto de política pública.

Contando con la ventaja que le brindaba el fuerte desendeudamiento previo, se llevaron adelante medidas para abordar el tratamiento de algunos temas postergados (CENDA, 2007) y enmarcarlos en un Plan "anticrisis". Entre las medidas más trascendentales pueden señalarse la "reestatización del Sistema Previsional Privado", el "Plan Argentina Trabaja" y la "Asignación Universal por Hijo", además de la consolidación institucional del SMVM como parámetro para la relación salarial, entre otros (Pastrana et al, 2012). Los resultados de las medidas fueron muy favorables durante un contexto internacional muy adverso, lográndose ciertos resultados de estabilidad en el plano laboral (Barrera Insua y Fernández Massi, 2017; Dabat, 2012).

\begin{tabular}{|c|c|c|c|c|}
\hline & 2008 & 2009 & 2010 & 2011 \\
\hline $\mathrm{PBI}$ & $4,1 \%$ & $-5,9 \%$ & $10,1 \%$ & $6,0 \%$ \\
\hline Tasa de actividad & $45,9 \%$ & $46,1 \%$ & $46,0 \%$ & $45,8 \%$ \\
\hline Tasa de empleo & $42,0 \%$ & $41,9 \%$ & $42,2 \%$ & $42,4 \%$ \\
\hline Tasa de desocupación & $8,4 \%$ & $9,1 \%$ & $8,3 \%$ & $7,4 \%$ \\
\hline Empleo no registrado & $37,0 \%$ & $36,0 \%$ & $35,2 \%$ & $34,3 \%$ \\
\hline
\end{tabular}

Fuente: Valores promedio. Encuesta Permanente de Hogares, INDEC.

Como vimos previamente, creció notablemente la cantidad de ocupados durante la postconvertibilidad; hasta el año 2011 se generaron cerca de cinco millones de empleos, lo que redujo notablemente el desempleo (representando menos del 7\% hacia fines de 2011). Un aspecto evidente tiene que ver con la tasa de actividad, que alcanzó máximos históricos durante este período. Sin embargo, luego del 2007 la economía argentina redujo su capacidad de generar puestos de trabajo; dándose también un cambio de tendencia a nivel sectorial, donde pasó a tener mayor peso el sector de servicios para generar puestos de empleo, que los vinculados a la producción de bienes (Santarcangelo y Perrone, 2013).

En materia salarial, entre los años de esta etapa el incremento del salario promedio fue superior al registrado en el SMVM, lo que refleja que las negociaciones paritarias se vieron favorecidas por toda la institucionalidad recuperada durante los primeros años de la postconvertibilidad, pero fuertemente condicionadas por el crecimiento de las presiones inflacionarias. Durante los años 2008 y 2009 se sostuvo en cierta medida la tendencia de recomposición salarial, con mayor fuerza en el sector privado, pero a partir de 2012 tendió a estancarse (Maito, 2019). 
Si bien se advierte durante todo el sub-período 2008-2011 una continuidad en la reducción del grado de informalidad, los niveles de empleo no registrado aún continuaron siendo importantes, impactando sobre un tercio de la población empleada.

A nivel agregado, durante el período 2003-2012 aumentó la productividad de la mano de obra, que no se dio por una extensión de la jornada de trabajo sino más bien por la intensidad del trabajo. En el período 2003-2007 su incremento estuvo dado en función del aumento de la escala gracias al aprovechamiento de la capacidad instalada ociosa. Luego del 2008 las posibilidades de incremento de la productividad giraron en torno a la posibilidad de lograr cambios tecnológicos y organizaciones, donde la producción creció y disminuyó la incorporación de nuevos trabajadores (Barrera Insua y Fernández Massi, 2017; Santarcangelo y Perrone, 2013).

\subsection{La sintonía fina del período 2012-2015}

Desde la salida de la convertibilidad y hasta fines del 2011 la dinámica de los indicadores económicos del país había sido prodigiosa. Con excepción del año 2009, en el cual el producto nacional acusó el impacto de la crisis internacional, la economía argentina siguió registrando aumentos del PIB del orden del 10\% y 6\% durante los años 2010 y 2011 respectivamente. En paralelo, tal como describen Coatz et al (2015), el sector industrial experimentó un crecimiento generalizado sustentado en la ampliación de la capacidad instalada (por encima del $5 \%$ ), importantes inversiones en bienes de capital, aumentos de productividad -aunque por debajo del aumento de salarios e impactando sobre la rentabilidady una fuerte demanda del mercado brasileño (principal destino de las exportaciones industriales). Este proceso permitió, asimismo, impulsar el crecimiento de la tasa de empleo. Sin embargo, hacia finales del período se hacía evidente que el modelo de acumulación vigente presentaba inconsistencias estructurales, requiriendo de manera impostergable la aplicación de políticas de "sintonía fina", tal como lo reconociera la misma Presidenta Cristina Fernández de Kirchner días después de haber obtenido un holgado triunfo electoral que aseguraba su segundo mandato (Casa Rosada, 2011).

Más allá de determinados matices de diagnóstico, existe un consenso bastante amplio en la literatura respecto a los resultados económicos de todo el período de la postconvertibilidad: si bien se generaron grandes avances vinculados a la recuperación de la industria, la creación del empleo, la mayor participación de los asalariados en la distribución del ingreso, el impulso a la ciencia y la tecnología, el nacimiento de nuevas PyMEs y la mejora de las condiciones sociales, Argentina siguió insertándose en la división internacional del trabajo principalmente como un proveedor de bienes intensivos en recursos naturales y de 
algunos pocos commodities industriales con escaso valor agregado y/o contenido tecnológico (Wainer, 2018; Fernández Bugna y Porta, 2007; Gerchunoff y Llach, 2018; Katz y Bernat, 2013; Hagman, 2014; Coatz et al; 2015).

El contexto internacional, lejos de mejorar desde el inicio de la crisis financiera, no daba señales alentadoras. Su impacto se vio reflejado tanto en la baja del precio de las commodities, principales productos exportados por Argentina, como en el estancamiento de la economía brasileña, principal socio comercial del país. Como consecuencia de ello, el frente externo se constituyó en un problema relevante. Con la implementación de controles de cambios y declaraciones juradas a la importación (DJAI) se buscó amortiguar su impacto. El escenario se completaba con instrumentos de sostenimiento de precios, como por ejemplo "precios cuidados", y financiamiento al consumo, en la línea de créditos "Ahora 12". Es decir, la estrategia giraba alrededor de protección de la oferta nacional y del estímulo al consuno, buscando impactar positivamente sobre el mercado interno.

\begin{tabular}{|c|c|c|c|c|}
\hline & 2012 & 2013 & 2014 & 2015 \\
\hline $\mathrm{PBI}$ & $-1,0 \%$ & $2,4 \%$ & $-2,5$ & 2,7 \\
\hline Tasa de actividad & $46,2 \%$ & $45,8 \%$ & $45,0 \%$ & $44,8 \%$ \\
\hline Tasa de empleo & $42,8 \%$ & $42,2 \%$ & $41,8 \%$ & $42,2 \%$ \\
\hline Tasa de desocupación & $7,2 \%$ & $7,9 \%$ & $7,1 \%$ & $5,9 \%$ \\
\hline Empleo no registrado & $34,4 \%$ & $33,7 \%$ & $33,5 \%$ & $32,5 \%$ \\
\hline
\end{tabular}

Fuente: Valores promedio. Encuesta Permanente de Hogares, INDEC.

Es decir, durante el período 2012-2015 las políticas implementadas desde el gobierno buscaban sostener y, en lo posible, reavivar el ciclo económico de post-convertibilidad. Mientras se procuraba mantener la capacidad de consumo, también se idearon políticas para alcanzar aspectos fundamentales para el desarrollo y el ascenso social, como el acceso a la vivienda y la educación superior en los sectores de medio y bajos ingresos (Gallo, 2017). En este sentido, los pilares para el sostenimiento de la capacidad de consumo giraron en torno al sostenimiento de toda la institucionalidad laboral construida en las etapas de postconvertibilidad previas, pero también mediante la constante actualización de los programas sociales y de protección social como la asignación universal por hijo y las jubilaciones. A su vez, en un país donde la mayoría de los proyectos se financian con recursos propios, y con el objetivo de brindar una señal financiera de mediano-largo plazo para toda la actividad económica, desde el gobierno se decidió implementar dos programas fondeados por la ANSES, el Ministerio de Economía y otros inversores privados mayoritarios: en 2012 se 
implementó la primera edición del Plan Procrear, que otorgaba créditos para la construcción de viviendas, y en 2014 se crea el Plan Progresar, que otorga beca de estudios para iniciar o finalizar la educación superior a los jóvenes desempleados o con empleos en condiciones de informalidad, siendo quienes habitualmente sufren mayor índice de desempleo y poseen menores ingresos (Trujillo, 2017).

Sin embargo, más allá de toda la batería de acciones y programas para reactivar la economía ${ }^{3}$, el nivel de generación de empleo se vio estancado. Desde la salida de la convertibilidad la participación del salario en el producto mostró un constante crecimiento, alcanzando su máximo en 2012, donde se mantuvo estancado en un valor promedio del 42,7\% hasta 2017, año en que comienza a descender (Kenedy et al, 2018; Maito, 2019; Santarcangelo y Perrone, 2013). Además, la reducción del trabajo no registrado morigeró sus progresos desde 2009 y durante el período $2012-2015$ se estancó alrededor del $34,4 \%$ y el $32,5 \%$, sosteniéndose un núcleo duro de informalidad que reacciona ante los estímulos de política pública. Un proceso similar se dio en la reducción de la desigualdad, que se detiene en 2012 y crece levemente en los años posteriores (Beccaria y Maurizio, 2017).

Evidentemente, más allá de los intentos desplegados desde el gobierno para mantener el ciclo económico, orientados a favor en la distribución de recursos a la clase trabajadora, la estructura productiva no respondió de la forma deseada. La dinámica de las cuentas macroeconómicas no favorecía a la competitividad de las firmas; la tendencia inflacionaria, y un tipo de cambio retrasado, tuvo su correlato en la balanza comercial, que comenzó a caer desde 2013, y en la profundización de la fuga de capitales. En este marco, el gobierno cerró acuerdos para cancelar deuda con el "Club de París" e indemnizar a Repsol por la reestatización de YPF, pero el fallo del juez Thomas Griesa terminó de empantanar el panorama financiero internacional para la Argentina, dando claras señales de una puja por ingresar a un nuevo ciclo económico, tendiente a favorecer en la distribución del ingreso al capital financiero.

\subsection{Vuelta al mercado. La situación durante un nuevo gobierno neoliberal}

Con el ascenso al poder de la Alianza Cambiemos, que proclamó a Maurico Macri como presidente de la República Argentina, se abrió una nueva etapa o ciclo en la economía nacional, que procuraba establecer una nueva estructura de distribución del ingreso. Ni bien asumió el nuevo gobierno, en diciembre de 2015 se levantaron las restricciones para la compra de moneda estadounidense, provocando una devaluación de más del $30 \%$ en un solo

\footnotetext{
${ }^{3}$ En 2014 se presenta el Plan Procreauto, que también otorgaba una línea de crédito para la
} compra de automóviles con el objetivo de reactivar la industria automotriz. 
día. El argumento de esta iniciativa era impulsar al ingreso de capitales extranjeros y estimular inversiones.

Además, se eliminaron los impuestos a las exportaciones de maíz y trigo, se redujeron los tributos de la soja y se removieron los gravámenes de comercio internacional en sectores específicos. Unas semanas después, en febrero de 2016, también se anunció la eliminación de retenciones para las mineras. Todo esto representó una importante reducción en la recaudación impositiva proveniente del comercio exterior. El argumento para estas medidas era promover el crecimiento del empleo en los sectores impositivamente favorecidos.

El panorama se completa con un cambio en el criterio respecto a las tarifas de los servicios de energía (gas y electricidad). De acuerdo a la información de la Secretaría de energía (Secretaría de Gobierno de Energía, 2019), el porcentaje de subsidios con relación al PIB fue marginal durante los primeros años de post-convertibilidad, comenzado a adquirir relevancia desde 2007 y a crecer sistemáticamente hasta 2014. Mientras el gobierno kirchnerista entendía a los subsidios como una oportunidad para estimular el consumo, mejorando indirectamente el salario real, la administración macrista fue mucho más pesimista al respecto y los entendía como uno de los causantes del déficit fiscal. Por ello, desde su asunción se fueron aplicando continuos ajustes (incrementos) en la tarifa de los servicios, denominándolo "sinceramiento" tarifario (que no fue otra cosa que la dolarización de las mismas). El efecto de esta política es la incapacidad de dar respuesta a un problema estructural de la economía argentina, la inflación. Lo mismo se observó con el precio de los combustibles, que siguieron la misma dinámica de ajuste que la política aplicada al tipo de cambio monetario. Podría señalarse que uno de los principales problemas de esta modificación de enfoque y política fue la ausencia de medidas compensatorias (Mastronardi y Mayer, 2015).

Entonces, a partir del escenario descripto, se puso en evidencia la existencia de una fuerte contradicción en la mirada que el gobierno tenía respecto de la forma de orientar la economía en general, y de resolver el problema de la inflación en particular. Desde un enfoque de "costos de producción", el gobierno consideraba que en las negociaciones paritarias se corría el riesgo de un mayor aumento de precios. Por ello, se dio un escenario de constante intromisión del gobierno para poner techo a las negociaciones colectivas de trabajo. En esa línea, tienen sentido las palabras del primer ministro de economía del gobierno de Macri, PratGay, que señaló que "cada sindicato sabrá dónde le aprieta el zapato y hasta qué punto puede arriesgar salarios a cambio de empleos" ("Prat-Gay y las paritarias", 2016, 1 de enero), en una particular alegoría a la Curva de Phillips. Sin embargo, luego aprobó aumentos en las tarifas de servicios, que generaron un nuevo ciclo inflacionario y deterioro del salario, dando 
como consecuencia una pérdida del $14,7 \%$ del poder adquisitivo del salario promedio de los trabajadores registrados durante el período 2016-2018, deterioro que fue aún mayor en el sector informal (Wahren et al, 2018).

\begin{tabular}{|c|c|c|c|c|c|c|c|c|c|}
\hline & 2011 & 2012 & 2013 & 2014 & 2015 & 2016 & 2017 & 2018 & 2019 \\
\hline $\begin{array}{c}\text { Inflación } \\
\text { (Ley de presupuesto) }\end{array}$ & 8,9 & 9,2 & 10,8 & 10,4 & 15,6 & 14,5 & 17 & 15,7 & 23 \\
\hline $\begin{array}{l}\text { Inflación anual } \\
\text { (INDEC) }\end{array}$ & 9,5 & 10,8 & 10,9 & 23,9 & 26,9 & 36,2 & 24,8 & $47,6 \%$ & 53,8 \\
\hline $\begin{array}{l}\text { Inflación anual (Índice } \\
\text { PriceStat - Promedio) }\end{array}$ & $24,8 \%$ & $25,8 \%$ & $20,9 \%$ & $38,5 \%$ & $27,2 \%$ & $35,2 \%$ & $23,7 \%$ & - & - \\
\hline $\begin{array}{c}\text { Tipo de cambio } \\
\text { BCRA (Peso/UDS) }\end{array}$ & \begin{tabular}{|c} 
Inicio: \\
3,98 \\
Fin: \\
4,30 \\
\end{tabular} & $\begin{array}{c}\text { Inicio: } \\
4,30 \\
\text { Fin: } \\
4,91 \\
\end{array}$ & $\begin{array}{c}\text { Inicio: } \\
4,92 \\
\text { Fin: } \\
6,51 \\
\end{array}$ & $\begin{array}{c}\text { Inicio: } \\
6,54 \\
\text { Fin: } \\
8,55\end{array}$ & $\begin{array}{c}\text { Inicio: } \\
8,55 \\
\text { Fin: } \\
12,97 \\
\end{array}$ & $\begin{array}{c}\text { Inicio: } \\
13,06 \\
\text { Fin: } \\
15,85\end{array}$ & $\begin{array}{c}\text { Inicio: } \\
\text { 15,91 } \\
\text { Fin: } \\
18,77 \\
\end{array}$ & $\begin{array}{c}\text { Inicio: } \\
\text { 18,55 } \\
\text { Fin: } \\
37,80\end{array}$ & $\begin{array}{c}\text { Inicio: } \\
37,93 \\
\text { Fin: } \\
59,89\end{array}$ \\
\hline
\end{tabular}

Fuente: Elaboración propia en base a Ley de presupuesto, INDEC, índice PriceStat y BCRA

Entonces, independientemente del cambio de paradigma en la forma de intervención estatal sobre la economía, los resultados registrados difícilmente permiten hablar de una intervención exitosa. La inflación se duplicó, mientras que la dinámica del tipo de cambio de la moneda norteamericana se modificó cualitativamente, con crecimientos que nada tienen que ver con el período anterior, pero sin generar cambios positivos en materia de comercio exterior, verificándose un estancamiento de las exportaciones. Al efecto propiciado por la devaluación, se sumó la eliminación de los derechos de exportación señalados previamente, y la eximición de la obligación de liquidar divisas en el mercado único y libre de cambios (MULC). Sin embargo, la intensidad de la apertura llevó al país a un déficit de la balanza comercial sin precedentes en 2017 y $2018^{4}$. El saldo comercial positivo recién llegaría en 2019 (USD 15.990 millones) debido a la caída en el nivel de actividad, que genera menor demanda de importaciones, además del encarecimiento de los productos importados por la fuerte devaluación del 2018.

Las políticas de corrección macroeconómicas implementadas durante la gestión del gobierno de Mauricio Macri tuvieron un fuerte impacto recesivo. Ello tuvo su correlato en los niveles de desempleo, que luego de registrar su menor valor en el tercer trimestre de 2015 ,

\footnotetext{
${ }^{4}$ En 2017 fue de USD 8.471 millones debido al incremento de las importaciones -en particular, de vehículos automotores, bienes de capital y bienes de consumo-. En 2018 la balanza comercial acumuló un déficit de USD 3.823 millones, un 54\% menor al rojo del año anterior, pero como resultado de una brutal devaluación (La más grande desde el fin de la convertibilidad. El peso se devaluó un 104\%) y la merma en la compra de bienes de capital, vehículos automotores, piezas y accesorios y bienes de consumo.
} 
fue creciendo hasta alcanzar valores de dos dígitos hacia finales del 2018. Según los informes técnicos del INDEC sobre "Mercado de trabajo. Tasas e indicadores socioeconómicos" (Instituto Nacional de Estadística y Censos [INDEC], 2018), en 2018 se incrementó el número de personas que, a pesar de estar ocupadas, se encontraban demandando empleo. Esto representaba un número cercano a los cuatro millones de argentinos. En otras palabras, no hubo recuperación del empleo en relación al 2017; peor aún, se redujo la cantidad de empleadores debido a la fuerte destrucción del aparato productivo durante este período (CEPA, 2020).

\begin{tabular}{c|c|c|c|c}
\hline \multicolumn{6}{c}{ Tabla 5. Evolución de la actividad, el empleo y la desocupación en } \\
Argentina (Años 2016-2019) \\
\hline & $\mathbf{2 0 1 6}$ & $\mathbf{2 0 1 7}$ & $\mathbf{2 0 1 8}$ & $\mathbf{2 0 1 9}$ \\
\hline PBI & $-2,1 \%$ & $2,8 \%$ & $-2,6 \%$ & $-2,1 \%$ \\
\hline Tasa de actividad & $45,8 \%$ & $45,9 \%$ & $46,6 \%$ & $47,3 \%$ \\
\hline Tasa de empleo & $41,9 \%$ & $42,1 \%$ & $42,3 \%$ & $42,6 \%$ \\
\hline Tasa de desocupación & $8,5 \%$ & $8,4 \%$ & $9,2 \%$ & $9,8 \%$ \\
\hline Empleo no registrado & $33,6 \%$ & $33,9 \%$ & $34,5 \%$ & $35,1 \%$ \\
\hline
\end{tabular}

Fuente: Encuesta Permanente de Hogares, INDEC.

El incremento del desempleo guarda relación con la caída en el uso de la capacidad instalada de la industria. Según el INDEC (INDEC, 2021), durante el período 2011-2015 se encontraba en un promedio del 73\%, mientras que en el bienio 2016-2017 cayó al 64,9\%, llegando incluso en 2019 a representar el 56,9\%. Basándonos en la información publicada por el Ministerio de Trabajo, Empleo y Seguridad Social, se observó una tendencia hacia el incremento de los trabajadores independientes, es decir monotributistas, lo que implica una clara señal respecto al proceso de flexibilización del mercado laboral. Este aspecto representa una pérdida de la calidad de empleo, además de la caída del ingreso promedio de los trabajadores. Durante el período 2016-2019 la participación del salario en el producto cayó desde el $42,8 \%$ al $37,4 \%$. 
Tabla 6. Trabajadores registrados según modalidad ocupacional principal. Con estacionalidad. Total país. En miles (Valores promedio. Años 2012 - 2018)

\begin{tabular}{|c|c|c|c|c|c|c|c|c|}
\hline Período & $\begin{array}{l}\text { Asalariados } \\
\text { privados }\end{array}$ & $\begin{array}{l}\text { Asalariados } \\
\text { públicos }\end{array}$ & $\begin{array}{l}\text { Asalariados } \\
\text { casas } \\
\text { particulares }\end{array}$ & 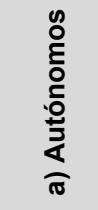 & 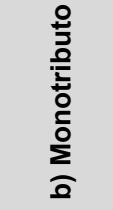 & 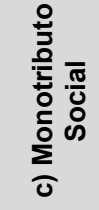 & $a+b+c$ & Total \\
\hline 2012 & $55,1 \%$ & $23,8 \%$ & $3,6 \%$ & $3,7 \%$ & $12,1 \%$ & $1,6 \%$ & $17,5 \%$ & 11009,0 \\
\hline 2013 & $53,6 \%$ & $24,5 \%$ & $3,8 \%$ & $3,7 \%$ & $12,4 \%$ & $2,0 \%$ & $18,1 \%$ & 11397,4 \\
\hline 2014 & $52,4 \%$ & $24,8 \%$ & $3,9 \%$ & $3,6 \%$ & $12,6 \%$ & $2,7 \%$ & $18,9 \%$ & 11668,7 \\
\hline 2015 & $52,3 \%$ & $25,4 \%$ & $3,7 \%$ & $3,4 \%$ & $12,1 \%$ & $3,0 \%$ & $18,5 \%$ & 11909,9 \\
\hline 2016 & $51,6 \%$ & $25,9 \%$ & $3,8 \%$ & $3,4 \%$ & $12,2 \%$ & $3,0 \%$ & $18,7 \%$ & 11990,0 \\
\hline 2017 & $51,1 \%$ & $25,8 \%$ & $3,8 \%$ & $3,3 \%$ & $12,7 \%$ & $3,3 \%$ & $19,3 \%$ & 12192,2 \\
\hline 2018 & $50,9 \%$ & $25,6 \%$ & $3,9 \%$ & $3,3 \%$ & $13,0 \%$ & $3,3 \%$ & $19,6 \%$ & 12306,2 \\
\hline 2019 & $49,5 \%$ & $26,7 \%$ & $4,1 \%$ & $3,3 \%$ & $13,4 \%$ & $3,0 \%$ & $19,7 \%$ & 12136,8 \\
\hline
\end{tabular}

Fuente: Elaboración propia en base a DGEMyEL-MPyT, Observatorio de Empleo y Dinámica Empresarial, sobre la base de los registros administrativos de los sistemas de la seguridad social (AFIP).

Al difícil panorama descripto, se le suman las malas decisiones en materia de política monetaria. Mientras que desde 2017 el tipo de cambio ingresó en una dinámica alcista, perjudicando a la balanza comercial, el gobierno sufrió un duro revés cuando el mercado financiero internacional nuevamente detuvo el crédito destinado hacia la Argentina, luego de que se emitiera deuda por USD 9.000 millones en 2018. El financiamiento del déficit externo y el endeudamiento no pueden ser ilimitados (Amico, 2020). La situación descripta se vuelve aún más preocupante si se considera el acuerdo cerrado por el gobierno de Mauricio Macri con el Fondo Monetario Internacional (FMI), lo que en el corto plazo podría implicar la imposición de recomendaciones basadas en los ya conocidos lineamientos del Consenso de Washington, que consisten básicamente en ajuste económico, que condiciona y repercute muy negativamente sobre el empleo y las jubilaciones, entre otros aspectos. En resumen, la deuda pública se incrementó en unos USD 100 mil millones en total durante el gobierno de Macri (Al 2015 la deuda ascendía a USD240.665 millones, alcanzando los USD 323.065 millones hacia fines de 2019 - Endeudamiento púbico con FMI y acreedores privados del exterior) (Ministerio de Economía, 2021). Ello condiciona fuertemente las posibilidades a futuro de asignar con libertad los recursos necesarios para implementar políticas de recomposición, crecimiento y desarrollo.

En cuanto a las PyMEs, que son la principal fuente de empleo de la economía argentina, lo que se perfilaba como un escenario positivo durante el gobierno 2011-2015, aún con sus 
restricciones o limitaciones, comenzó a cambiar preocupantemente de signo a partir del período iniciado en 2016, conforme se fue estructurando el nuevo esquema de política pública nacional descripto previamente.

La dinámica y potencia de las ventas comenzó a evidenciar señales desalentadoras, conforme al menor crecimiento de la demanda interna y a la menor compra de los socios en el mercado regional, especialmente desde Brasil. Esta menor performance de facturación, comienza a resentir el flujo de caja de las PyMEs y se afecta la capacidad de generar recursos propios, para destinar a proyectos de mediano plazo, como inversión e innovación, entre otros.

La atmósfera se agrava aún más, en la medida que se endurecen las condiciones del crédito local, tanto por fuerte reducción de la oferta por parte de entidades públicas (menor cantidad de líneas de crédito bancario y reducción drástica de los instrumentos de promoción), como así también por el aumento de la tasa de referencia del Banco Central de la República Argentina, que fijó un piso básico del orden del 30\%, para el rendimiento de productos financieros. Este esquema de contracción monetaria complejizó notablemente el acceso y repago del crédito comercial y gravó de modo importante el proceso de redescuento de cheques para capital de trabajo. A todo ello debe sumarse el escenario aún más complejo que afrontaron por el notable encarecimiento de sus estructuras de costos, vía incremento de las tarifas de servicios públicos y el aumento sostenido en los gastos por provisión de combustibles. En suma, las políticas implementadas durante el gobierno macrista, no solo afectaron al empleo de manera directa, sino también por el lado del endurecimiento de las condiciones de funcionamiento de las empresas que emplean el mayor número de trabajadores, las PyMEs.

Finalmente, es importante mencionar que el gobierno terminó sin poder aplicar un proyecto de flexibilización laboral, imposibilitado por el negativo resultado de las elecciones presidenciales de 2019. En este punto, si bien es cierto que se observa desactualización en los convenios colectivos de trabajo de un importante número de sectores, además del surgimiento de un conjunto de nuevas actividades que funcionan sin ningún tipo de regulación (como por ejemplo las actividades relacionadas con plataformas digitales de servicios $y$ delivery), el abordaje de esta cuestión requiere de un espíritu pragmático, que defina nuevas formas institucionales acordes a las necesidades de cada sector y al contexto del mercado dónde se desenvuelven.

\section{Conclusión}


Sin posibilidad de respiro, al inicio de la gestión del gobierno de Alberto Fernández, los desafíos que se debía abordar en materia económica resultaban sumamente complejos. A la preocupante recesión económica se le sumó la presión de una deuda externa imposible de afrontar en el corto plazo. La economía argentina se ve urgida de generar divisas para afrontar los compromisos de deuda contraídos durante la segunda parte del gobierno de Mauricio Macri. Entonces, ante la imposibilidad de recurrir a fuentes financieras externas, las expectativas en materia de comercio exterior pueden resultar excesivas, sobre todo si se tiene en cuenta la dinámica comercial reciente.

De acuerdo con el marco teórico planteado al inicio de este trabajo, resulta necesaria la definición de formas institucionales que regulen el inicio de un nuevo ciclo económico. El intento de implementar un modelo de desarrollo basado en la hipótesis neoclásica de distribución del ingreso, como la implementada por el gobierno macrista, fracasó nuevamente en Argentina. En este sentido, una vuelta a la hipótesis keynesiana, con intervención estatal y estímulo al desarrollo de la capacidad competitiva del entramado productivo local parece ser un camino más adecuado. En esta línea es importante generar las condiciones macroeconómicas más adecuadas para el establecimiento de un positivo ambiente de negocios.

En materia de intervención estatal, previo al impacto de la pandemia COVID-19, se direccionaron esfuerzos con miras a estimular el consumo interno, como el aumento salarial a estatales y privados a principio de 2020, y el control de precios mediante el relanzamiento del "programa Precios Cuidados". Para la oferta, moratoria fiscal para PyMEs, reducción de tasa de interés y líneas de crédito para alivianar financieramente a las empresas de menor tamaño. Si se considera la elevada capacidad ociosa, con la que operan las firmas que sobrevivieron a las políticas económicas del modelo anterior, es posible lograr una reactivación económica relativamente rápida. Sin embargo, el principal desafío será sostenerla en el tiempo. En este sentido, también genera optimismo el incremento de recursos anunciados para el área científico-tecnológica, que se materializan en el incremento de becas del CONICET y recursos para la ANPCyT.

En cuanto a la oferta exportable de la Argentina, la principal dificultad radica en la fuerte dependencia por los productos primarios. Se depende mucho de la producción "agro", sector que ya ha marcado la cancha con reclamos por la simple actualización del esquema tributario del gobierno anterior, lo que reduce las expectativas de aportes de divisas de parte de este sector, y condiciona mucho la aplicación de reformas de carácter estructural. Por ello, el desafío es ampliar la participación de la industria (y de otros sectores de importante 
dinamismo comercial, como los bienes y servicios intensivos en conocimiento) en el comercio exterior, para lograr mayor grado de diversificación en la oferta.

El desarrollo de la industria resulta crucial para impulsar un nuevo ciclo de crecimiento, pero se requiere de una transformación muy profunda para sustituir la importación de insumos intermedios por producción nacional y alcanzar el desarrollo de una oferta exportable. Resultará de suma importancia coordinar misiones y acuerdos comerciales, pero también lograr un salto de calidad en los bienes y servicios producidos localmente (con mayor incorporación de contenido tecnológico). Los desafíos son bien conocidos: mayor eslabonamiento productivo hacia el interior del territorio nacional; lograr inserción en cadenas globales de valor, apuntando a las funciones que generan mayor valor agregado, como I+D, diseño, y posventa; y avanzar en el desarrollo de exportaciones de intangibles, como por ejemplo biotecnología y software, entre muchos otros.

A lo largo del trabajo se ha observado cómo durante los tres gobiernos kirchneristas se construyó toda una institucionalidad para la recuperación de la fuerza de trabajo de la flexibilización ejercida durante la convertibilidad. Si bien las acciones desplegadas fueron insuficientes para incorporar a una mayor porción de la fuerza de trabajo dentro del mercado formal, resulta muy evidente todo el recorrido y el saldo positivo respecto del ciclo económico anterior, no sólo en materia salarial, sino también en el fortalecimiento de los sindicatos y de las instancias de negociación paritaria. Sin embargo, todo ello es insuficiente si las condiciones macroeconómicas no favorecen a la generación de un adecuado ambiente de negocios. El problema es que muchas veces se entiende que el camino para lograr un buen ambiente de negocios es mediante la reducción de salarios.

Es posible considerar que gran parte de la organización sindical, reconstruida durante las primeras etapas de post-convertibilidad, permitieron mantener buena parte de la institucionalidad laboral frente a las políticas de flexibilización y reducción salarial del gobierno macrista, pero no es suficiente para la construcción de un nuevo ciclo de crecimiento económico. Es vital frenar la inflación porque ello deteriora el salario real, pero igual de importante es la actualización de las habilidades y calificación de la fuerza de trabajo. El avance de la tecnología condiciona fuertemente a la inserción laboral, pero también evidencia la necesidad de implementar acciones para la reconversión y actualización de la fuerza de trabajo. En Argentina se cuenta con instituciones educativas y de generación de conocimiento sumamente prestigiosas. Además, ANSES se ha consolidado como una institución con la información necesaria y la capacidad para llegar a los sectores que mayor ayuda requieren. Finalmente, es importante definir una estrategia de acción coordinada entre los niveles de gobierno, los sindicatos y el sector empresarial. 
La pandemia COVID-19 ha quitado de la agenda el proyecto de flexibilización laboral macrista, que buscaba legitimarse bajo argumentos que cargaban las tintas sobre la desactualización de los convenios colectivos de trabajo. Quizás resulte conveniente no dejar pasar la oportunidad de retomar la agenda de actualización de los convenios colectivos de trabajo, para ajustarla a las necesidades de actualización de la clase trabajadora, y construir una posición táctica favorable para tratar la cuestión de forma pro-actividad, evitando una situación donde la posición del trabajador se encuentre a la defensiva.

\section{Referencias bibliográficas}

Abeles, M. (2009). El impacto de la crisis internacional en la economía argentina. Revista de Trabajo 5(7), pp. 185-212.

Amico, F. (2020). La macroeconomía de Macri: Adiós represión financiera, bienvenido nuevo default. Circus - Revista Argentina de Economía 5(7), pp. 52-89.

Anigstein, C. (2012). La revitalización de la negociación colectiva en Argentina y sus controversias. VII Jornadas de Sociología, UNGS, San Miguel. Recuperado de: https://www.ungs.edu.ar/wp-content/uploads/2012/11/gt2 mesa-3 Anigstein.pdf

Astarita, R. (2018). Keynes, poskeynesianos y keynesianos neoclásicos: Apuntes de economía política ( $2^{\mathrm{a}}$ ed. Ampliada). Bernal: Universidad Nacional de Quilmes.

Barrera Insua, F. \& Fernández Massi, M. (2017). La dinámica productiva como límite superior de los salarios en la industria argentina. Revista Perfiles Latinoamericanos (FLACSO México) 25 (50), pp. 301-329.

Beccaria, L. \& Maurizio, R. (2017). Mercado de trabajo y desigualdad en la Argentina. Un balance de las últimas tres décadas. Sociedad [En línea], 37, pp. 15-41.

Beccaria, L. (2007). El mercado de trabajo luego de la crisis. Avances y desafíos. En: Kosacoff, B. (ed.). Crisis, recuperación y nuevos dilemas. La economía argentina 2002-2007. Santiago de Chile: CEPAL, Naciones Unidas.

Boyer, R. (1992). La teoría de la regulación: un análisis crítico. Valencia: Edicions Alfons el Magnánim/IVEI.

Brenner, R. (1999). El desarrollo desigual y la larga fase descendente: Las economías capitalistas avanzadas desde el boom al estancamiento, 1950-1998. Encuentro XXI, 14. 
CENDA (2007). Notas de la economía argentina. Informe Macroeconómico $n^{\circ} 4$. Centro de Estudios para el Desarrollo Argentino, Buenos Aires.

CEPA (2020). Análisis de la dinámica laboral y empresarial (2015-2020). Evolución de los principales indicadores durante la gestión de Cambiemos y los meses de impacto de la pandemia COVID-19. Informe del Centro de Economía Política Argentina (ISSN 2796-7166). Buenos Aires. Recuperado de: https://centrocepa.com.ar/informes/265-analisis-de-ladinamica-laboral-y-empresarial-2015-2020-evolucion-de-los-principales-indicadores-durantela-gestion-de-cambiemos-y-los-meses-de-impacto-de-la-pandemia-del-covid-19.html

Chamorro, A. (2011). Algunos elementos sobre la teoría clásica del empleo y la versión keynesiana. Tendencias. Revista de la Facultad de Ciencias Económicas y Administrativas. XII(2), pp. 35-57.

Coatz, D., Grasso, F. \& Kosacoff, B. (2015). Desarrollo industrial: recuperación, freno y desafíos para el desarrollo en el siglo XXI. EDICON, Fondo Editorial Consejo, Consejo Profesional de Ciencias Económicas de la Ciudad Autónoma de Buenos Aires, Ciudad Autónoma de Buenos Aires.

Costa, A., Langer, A. \& Rodriguez J. (2003). Fundamentos de Economía. Buenos Aires: Ediciones Cooperativas.

Dabat, A. (2012). El rumbo de la economía argentina bajo el kirchnerismo. Economía UNAM, 9(26), pp. 43-67.

Delfini, M. (2016). Determinantes de la precarización laboral en Argentina entre 2003-2013: entre los cambios y las continuidades. Investigación \& Desarrollo 24(1), pp. 53-75.

Prat-Gay y las paritarias: "Cada gremio sabrá hasta qué punto puede arriesgar salarios a cambio de empleos". (2016, 1 de enero). El Cronista. Recuperado de: https://www.cronista.com/economiapolitica/Prat-Gay-y-las-paritarias-Cada-gremio-sabra-hastaque-punto-puede-arriesgar-salarios-a-cambio-de-empleos-20160101-0005.html

Fernández Bugna, C. \& Porta, F. (2007). El crecimiento reciente de la industria argentina. Nuevo régimen sin cambio estructural. En: Kosacoff, B. (ed.). Crisis, recuperación y nuevos dilemas. La economía argentina 2002-2007. Santiago de Chile: CEPAL, Naciones Unidas.

Gajst, N. (2010). La escuela francesa de la regulación: Una revisión crítica. Revista Científica "Visión de Futuro" 13(1).

Gallo, M. (2017). La economía argentina durante el período 2004-2017: ciclo expansivo, restricción externa y retorno de la valorización financiera, Comunicación presentada en el II 
Congreso de Economía Política para la Argentina; Universidad Nacional de Avellaneda (UNDAV), Buenos Aires. Recuperado de: http://nulan.mdp.edu.ar/2757/1/gallo-2017.pdf

Gerchunoff, P. \& Llach, L. (2018). El ciclo de la ilusión y el desencanto: un siglo de políticas económicas argentinas de 1880 a nuestros días. Ciudad Autónoma de Buenos Aires: Editorial Crítica.

Gómez, M. (1997). Conflictividad laboral durante el Plan de Convertibilidad en Argentina (1991-1995). Las prácticas de la lucha sindical en una etapa de reestructuración económica y desregulación del mercado de trabajo. Estudios sociológicos XV (45), pp. 639-689.

Guido, P. (2010). Análisis del proyecto de ley del presupuesto 2011. La política presupuestaria del kirchnerismo, en DOCUMENTOS del Centro para la Apertura y el Desarrollo de América Latina (CADAL), Año VIII Número 114. Buenos Aires. Recuperado de: https://www.cadal.org/documentos/documento_114.pdf

Hagman, I. (2014). La Argentina Kirchnerista en tres etapas: una mirada crítica desde la izquierda popular (1a ed.). Ciudad Autónoma de Buenos Aires: Cuadernos de cambio, Patria Grande.

Instituto Nacional de Estadística y Censos, INDEC. (2018). Mercado de trabajo. Tasas e indicadores socioeconómicos (EPH). Informes Técnicos, 2 (178). Recuperado de: https://www.indec.gob.ar/uploads/informesdeprensa/mercado trabajo eph 2trim18.pdf

Instituto Nacional de Estadística y Censos, INDEC. (2021). Utilización de la capacidad instalada en la industria. Informes Técnicos, 5 (21). Recuperado de: https://www.indec.gob.ar/uploads/informesdeprensa/capacidad 02 214ABA20231A.pdf

Jofré, J. \& Ferreyra, M.A. (2010). Elementos regulacionistas para una aproximación al ordenamiento territorial. Revista Digital Proyección, 8. Recuperado de: https://bdigital.uncuyo.edu.ar/objetos digitales/11374/02-proyeccion8-jofre.pdf

Katz, J. \& Bernat, G. (2013). Interacciones entre la macro y la micro en la post convertibilidad: dinámica industrial y restricción externa. Desarrollo Económico 52(207-208), pp. 383-404.

Kenedy, D., Pacífico, L. \& Sánchez, M. (2018). La masa salarial y su composición según el vínculo laboral. Argentina. 1993-2017: propuesta de estimación en el marco de la base 2004 (2005-2015) y empalme con la base 1993. Doc. de trabajo / Informes N²4, CEPED-UBA. 1a ed. - Ciudad Autónoma de Buenos Aires: Universidad de Buenos Aires. Facultad de Ciencias Económicas. Recuperado de: http://biblioteca.clacso.edu.ar/Argentina/ceped$\underline{\text { uba/20200511085551/24.pdf }}$ 
Lindemboim, J. (2001). El deterioro del mercado de trabajo y las "nuevas" relaciones laborales. Revista Enoikos IX (18).

Lozano, C. (2014). Análisis del Presupuesto 2015. Otro presupuesto mentiroso, deficitario y endeudador, en Instituto Pensamiento y Políticas Públicas (IPyPP). Buenos Aires. Recuperado de: https://ipypp.org.ar/descargas/2014/EL\%20PRESUPUESTO\%202015.pdf

Maito, E. (2019). La distribución funcional del ingreso en Argentina (1993-2016). Trabajo y Sociedad 32, pp. 53-78.

Marticorena, C. (2005). Precariedad laboral y caída salarial. El mercado de trabajo en la Argentina post convertibilidad, $7^{\circ}$ Congreso Nacional de Estudios del Trabajo: Nuevos escenarios en el mundo del trabajo: rupturas y continuidades, Asociación Argentina de Especialistas en Estudios del Trabajo (ASET), Buenos Aires, 10 al 12 de agosto de 2005.

Mastronardi, L. \& Mayer, M. (2015). Quita de subsidios a la energía en Argentina: Análisis de bienestar mediante un MEGC. Estudios Económicos XXXII (65), pp. 47-71. Recuperado de: https://ri.conicet.gov.ar/bitstream/handle/11336/71179/CONICET Digital Nro.7e17f830-57654b6f-a15c-60f036ebf026 B.pdf?sequence=5\&isAllowed=y

Ministerio de Economía (Finanzas), Presidencia de la Nación Argentina. (2021). Presentación gráfica de la deuda. Recuperado de: https://www.argentina.gob.ar/economia/finanzas/presentaciongraficadeudapublica

Neffa, J. (2012). La evolución de la relación salarial durante la post convertibilidad. Revue de la régulation. Capitalisme, institutions, pouvoirs (En linea), 11. Recuperado de: http://journals.openedition.org/regulation/9695

Nicholson, W. (2008). Teoría microeconómica. Principios básicos y ampliaciones (9na ed.). México: Cengage Learning Editores, S.A.

Pastrana, F., Toledo, F., \& Villafañe, S. (2012). El modelo económico ante la crisis internacional: sostenimiento de las condiciones laborales y sociales en Argentina. En Macroeconomía, empleo e ingresos: debates y políticas en Argentina frente a la crisis internacional 2008-2009. Buenos Aires: Ministerio de Trabajo, Empleo y Seguridad Social, y Oficina de País de la OIT para la Argentina. Recuperado de: https://www.argentina.gob.ar/sites/default/files/macroeconomia empleo e ingresos.pdf

Pérez, P. \& Neffa, J.C. (2006). La teoría general del empleo según J. M. Keynes. En Teorías económicas sobre mercado de trabajo I (pp. 105-134). Buenos Aires: Ed. Fondo de Cultura Económica/CEII-PIETTE/Trabajo y Sociedad. 
Porta, F. \& Bianco, C. (2011). El vínculo entre la distribución del ingreso y acumulación. Revisión teórica y prescripciones de política. En Novick, M y Villafañe, S (Eds.). Distribución del ingreso. Enfoques y políticas públicas desde el sur (1a ed.). Buenos Aires: PNUD Ministerio de Empleo, Trabajo y Seguridad Social.

Santarcangelo, J., \& Perrone, G. (2013). El desempeño del mercado de trabajo argentino durante la postconvertibilidad. Ensayos de Economía 23(43), pp. 45-61.

Secretaría de Gobierno de Energía, Ministerio de Hacienda, Presidencia de la Nación Argentina (2019). Evolución de subsidios, oferta y demanda de energía 2015-2019: Gas, electricidad y petróleo. Recuperado de: http://www.energia.gob.ar/contenidos/archivos/Reorganizacion/sintesis balance/2019-1120 SE Subsidios oferta y demanda de energia Argentina 2015-2019 dist.pdf

Trujillo, L. (2017). La Argentina kirchnerista: Alcances y límites de una experiencia democrática sobre la distribución del ingreso (2003-2015). Polis [En línea] 46, pp. 1-24. Recuperado de: http://journals.openedition.org/polis/12289

Wahren, P., Harracá, M. y Cappa, A. (2018). A tres años de Macri: Balances y perspectivas de la economía argentina. Buenos Aires: CELAG. Recuperado de: https://www.celag.org/wpcontent/uploads/2018/12/A TRES AN\%CC\%830S DE MACRI BALANCES Y.pdf

Wainer, A. (2018). Economía y política en la Argentina kirchnerista (2003-2015). Revista Mexicana de Sociología 80(2), pp. 323-351. 